\title{
Habilidades y desempeños: rutas para la formación en investigación del militar ${ }^{1}$
}

https://doi.org/10.21830/9789585241459.02

\author{
Juan María Cuevas Silva \\ Escuela de Armas Combinadas del Ejército
}

\section{Resumen}

Antecedentes: la formación del militar debe estar acompañada de procesos curriculares orientados a fortalecer, dinamizar y potencializar las competencias, las habilidades y los desempeños de las personas que se forman en este contexto. Objetivos: este escrito hace un acercamiento a las habilidades, competencias y desempeños que se consideran pertinentes, relevantes y necesarios para responder a los retos de una formación militar y que, a su vez, está inmersa en procesos sociales que exigen la presencia de un militar con habilidades blandas y duras, así como desempeños con responsabilidad social. Materiales y métodos: se realizó una búsqueda en bases de datos sobre investigaciones o artículos que aborden el tema de las habilidades blandas y duras. Resultados: se determina una serie de habilidades y desempeños acordes con la educación formal y, específicamente, para la educación o formación militar. Conclusiones: una de las principales conclusiones de este análisis documental es que se debe formar a los militares en habilidades blandas, enmarcadas en políticas y desarrollos de los procesos sociales, que requieren de un militar que tenga la capacidad de transformar situaciones en oportunidades de cambio y mejora de la sociedad.

Palabras clave: competencias; competencias militares; desempeños; formación militar; habilidades.

1 Capítulo de libro que presenta parte de los resultados del proyecto de investigación Práctica Pedagógica en el Ejército Nacional: Reflexiones en torno a la Formación y la Investigación, del Grupo de Investigación para la Capacitación Militar (GICAM) de la Escuela de Armas Combinadas (ESACE), registrado con el código COL0160714 de Minciencias. Los puntos de vista y los resultados de este capítulo pertenecen al autor y no reflejan necesariamente los de las instituciones participantes.

2 Doctor en Procesos Sociales y Políticos en América Latina de la Universidad Arcis de Santiago de Chile. Magíster en Educación de la Universidad Pedagógica Nacional. Licenciado en Filosofía de la Universidad de San Buenaventura. Profesor de Metodología de la Investigación en la ESACE. Profesor Asociado de la UMNG. Miembro de la Mesa de Formación en ética de la investigación, bioética e integridad científica de Minciencias. Orcid: https://orcid.org/0000-0002-1680-6223. Contacto: juan.cuevassilva@cedoc.edu.co 


\section{Introducción}

El mundo cambiante que caracteriza nuestra época y sociedad exige transformar la educación hacia el desarrollo de habilidades, competencias y desempeños que permitan a los estudiantes transferir lo que aprenden en el aula a múltiples contextos, incluyendo las situaciones que aún desconocemos y que se pueden presentar en el futuro próximo. Por esta razón es indispensable reflexionar sobre las habilidades, las competencias y los desempeńos que los estudiantes militares desarrollan en sus estudios, en cuanto condición necesaria para plantear un proyecto de formación para la investigación que les permita acercarse a la solución de problemas y/o a la transformación de situaciones, de tal manera que aumente el impacto positivo que tienen en la sociedad como militares. Sin embargo, formar a un militar con una base curricular centrada en la investigación va más allá de que formule proyectos, pues además es fundamental que sepa leer los contextos y las realidades que son cobijadas por procesos sociales, que identifique los nichos de problemas, los hechos, las circunstancias y las eventualidades a las cuales debe saber dar respuesta con sus habilidades, competencias y desempeños.

En este sentido, la educación militar en Colombia ha estado a la vanguardia de las exigencias que plantean los procesos sociales, los cuales han generado un cambio educativo significativo: el proceso enseñanza-aprendizaje se aborda ahora desde una visión holística que reconoce la relevancia del aprender a aprender, aprender a hacer, aprender a ser, entre otras tendencias pedagógicas, como la formación para la resolución de problemas. No obstante esta transformación, los avances de la sociedad y los modelos de organización de la ciudadanía hacen necesario que la educación propicie el desarrollo de habilidades propias para la investigación, pues desde allí se cultiva el progreso y el desarrollo de una sociedad.

De acuerdo con lo anterior, este capítulo analiza las características de la educación en investigación que tienen los militares en diferentes instituciones de formación, así como las habilidades que prioriza la perspectiva curricular. Del mismo modo, se establece cuáles son las habilidades necesarias para formar a estos estudiantes como investigadores (no necesariamente científicos) y prepararlos para desarrollar investigaciones no solo exitosas, sino también valiosas para la sociedad a la cual sirven. Finalmente, se revisan cuáles son las mejores aproximaciones pedagógicas para alcanzar el desarrollo exitoso de esas habilidades y, más importante aún, su transferencia hacia otros contextos fuera de las aulas de clase. 


\section{Habilidades para la investigación en la educación}

La formación del militar se ha caracterizado porque se acopla con los modelos políticos implantados en los Estados. El sistema político actual llamado democracia cimenta sus bases en la concepción del ser humano como ciudadano perteneciente a un país, Estado, república y nación. En este contexto político, la instancia militar tiene la responsabilidad de defender la nación. Sin embargo, es necesario preguntarse si sus miembros se forman como militares o ciudadanos, como plantea Máximo Badaró (2009). En la investigación que hace sobre la sociedad militar y su educación en Militares o ciudadanos. La formación de los oficiales del ejército Argentino, el autor muestra aspectos relevantes de la forma como el militar argentino ha tenido que acoplarse y sincronizarse con los procesos de transformación de la sociedad argentina. Este tipo de estudios evidencian que la formación militar sí ha realizado cambios profundos en su sistema, de manera que ha dejado de ser meramente instruccional y ha logrado responder a las exigencias de la sociedad. Particularmente, el trabajo de Badaró (2009) constituye un acercamiento al significado de las habilidades y el rigor en su desarrollo que demanda la formación militar.

\section{Habilidades complejas y simples}

La formación para la investigación requiere un currículo donde se planteen e incluyan habilidades que se deben potenciar para el quehacer del investigador y cuyo aprendizaje y desarrollo necesitan contextos adecuados. Así mismo, debe construir estrategias pedagógicas y didácticas que no solamente dinamicen y potencien estas habilidades, sino que además sean acordes con un proyecto curricular que diferencie claramente las habilidades complejas y las habilidades sencillas.

Para comprender con mayor profundidad esta diferenciación, Shafer (2016) plantea que las habilidades pueden variar de acuerdo con el grado de complejidad que las caracterice. Así, existen habilidades simples y complejas: las simples son aquellas que no pueden ser divididas entre diferentes habilidades más pequeñas. Por ejemplo, recordar, conectar, prestar atención, escuchar activamente, etc. Por su parte, las habilidades complejas están compuestas por diferentes habilidades simples, por ejemplo el autocontrol, que implica habilidades como la atención, la predicción (comprensión de causa-efecto), la conexión, etc.

Las habilidades simples están directamente relacionadas con una dimensión cognitiva, con una sola funcionalidad que le permite al sujeto entrar en contacto con el entorno gracias a sus procesos psicosensoriales. Por su puesto, este proceso de interacción ocurre en el cerebro, donde se construyen las habilidades complejas, 
que se pueden descomponer en sus dimensiones psicosensoriales y cognitivas, y que permiten aprehender y construir conocimiento. Es necesario aclarar que las habilidades están directamente relacionadas con las capacidades físicas, de tal forma que el cuerpo y sus connotaciones genotípicas y biológicas son indispensables para su desarrollo, ya sean simples o complejas. Esto no quiere decir que una persona que tenga algún tipo de limitación física no pueda desarrollar habilidades; por el contrario, incluso puede desarrollar habilidades complejas que una persona con todas sus facultades físicas no alcanza a desarrollar.

Reconocer que existen estos dos tipos de habilidades permite escalonar el aprendizaje y, por tanto, los objetivos que se definen en el diseńo curricular, la planificación, la ejecución y la evaluación en un contexto educativo con el fin de alcanzar las habilidades simples y complejas que se pretende desarrollen los estudiantes. Así, volviendo a un ejemplo anterior, si se establece como objetivo final que el estudiante desarrolle el autocontrol, entonces habrá que plantear estrategias y tareas para que adquiera las habilidades simples que la componen. Esto permite entender el proceso escalonado del aprendizaje y plantear procesos adecuados para que los estudiantes desarrollen las habilidades que se establecen en el currículo. En el ejemplo propuesto, para que una persona desarrolle la habilidad compleja de autocontrol debe tener habilidades sencillas como la atención, la concentración, el saber ver y escuchar, así como saber ubicarse en el contexto. Aún más, el autocontrol implica además la toma de decisiones, que también se considera una habilidad compleja.

Ahora bien, no basta con desarrollar habilidades sencillas y complejas, sino que además es necesario tener en cuenta las habilidades duras y blandas, especialmente si se trata de la formación militar.

\section{Habilidades duras y blandas}

Las habilidades complejas y simples se pueden clasificar en las categorías tradicionales de habilidades duras y blandas. Las primeras son aquellas que se pueden evaluar fácilmente a través de los métodos tradicionales de evaluación sumativa y que se enfatizan en el diseño curricular de la mayoría de los programas de educación básica, secundaria y superior, ya que se pueden observar y evaluar con mayor facilidad. Ejemplos de habilidades duras son la lectura, la escritura, el análisis económico, el diseño estratégico, etc.

Como elementos de análisis, a continuación se presentan distintas definiciones de habilidades duras y blandas. Sobre las primeras se encuentran las siguientes acepciones: 
- La psicología sobre las habilidades cognitivas o duras define la habilidad cognitiva como "la habilidad o aptitud para la percepción, el aprendizaje, la memoria, la comprensión, la conciencia, el razonamiento, la intuición, el juicio y el lenguaje". El coeficiente intelectual, por su parte, se refiere a los resultados de las pruebas de inteligencia (Bassi et al., 2012, p. 82).

- Se considera que las habilidades duras (conocimientos teóricos) se aprenden de manera más rápida que las habilidades blandas, ya que éstas últimas están íntimamente relacionadas por los rasgos de personalidad, es por esta razón que son más difíciles de enseñar. El contexto en el cual está inserta la persona es fundamental al momento de observar el desarrollo de estas habilidades en los sujetos (Lagos, 2012).

- Por sus características, esta prueba de habilidad constituye una medida "dura" u objetiva y sus resultados se expresan como el número de analogías correcta (BID, 2012, pp. 97-112).

- Habilidades duras o hard skills: parte de un coeficiente intelectual de una persona y tiene que ver con la capacidad para realizar un determinado tipo de terea o actividad; están vinculadas al conocimiento, a lo mesurable en términos exactos, a destrezas puntuales. Por ejemplo, dominar el inglés en nivel avanzado. Se puede medir y calificar (Roca, 2015).

- Las habilidades y herramientas técnicas o "habilidades duras" solo dan respuesta a escenarios ya probados y conocidos; y no permiten enfrentar problemas en escenarios de incertidumbre, donde las respuestas no son conocidas. Por ende, llevan a cuestionar, no solo la actual manera de proceder en una organización, sino que llevan a cuestionar los valores, actitudes y hábitos. Las llamadas "habilidades duras" son de índole técnica y son elementos básicos que no generan mayor diferenciación, ni tampoco agregan valor. Por el contrario, las "habilidades blandas", son las que hoy, hacen la verdadera diferencia entre un profesional y otro. Son a su vez, las que realmente agregan valor a una organización (Arroyo, 2012, pp. 35-36).

En segundo lugar, respecto a las habilidades blandas se encuentran las siguientes definiciones (Chávez, 2016):

- Se entenderá como habilidades blandas "a un conjunto de habilidades no-cognitivas esenciales para aprender y desempeñarse exitosamente en el trabajo". Estas habilidades son llamadas también del "saber ser", es 
decir, cómo debe ser una persona, qué habilidades debe poseer para desarrollar actividades y para relacionarse con los demás.

- Saber ser: consiste en saberse relacionar consigo mismo, los demás y el entorno con optimismo, espíritu de reto, proyección y buen trato, está compuesto por instrumentos emocionales (responsabilidad), estrategias afectivas (delicadeza, honradez) y estrategias sociales (trabajo en equipo), de ahí se puede decir que el saber ser practica la autorreflexión para mejorar el desempeño (Tobón, 2009, p. 174).

- Son aquellas orientadas al desarrollo de aptitudes sociales. Son reconocidas de mejor manera por los empleadores cuando un estudiante, joven o trabajador busca empleo. Se trata de capacidades comunicativas, de trabajo en equipo, flexibilidad y adaptabilidad frente a un determinado trabajo, entre otras (Valdevenito, 2013).

- Habilidades blandas son esencialmente habilidades personales, las no técnicas, intangibles, habilidades personales específicas que determina la fuerza de uno como un líder, escuchador, negociador, y mediador de conflicto. Habilidades blandas es un término que refiere a los rasgos personales, gracias sociales, facilidades de habla, hábitos personales, amabilidad, y optimismo que marca a las personas en variedad de grados. (Alex, 2016, p. 2).

- Habilidades o competencias blandas: son las referentes a las habilidades sociales, la capacidad de comprensión del otro, la dimensión de relaciones intersubjetivas, relacionadas con la inteligencia emocional y la dimensión afectiva de la persona. (Mujica, 2015).

- Son las que han estado más ocultas —e incluso discriminadas - en los entornos laborales, pero son las que están resurgiendo con más fuerza. ¿Por qué? Porque cuando hablamos de pow (presfut of work) hablamos de colaboración, de proyectos colaborativos, de entornos laborales donde prima lo social (social business, social network) y se trabaja en jerarquía. $\mathrm{Y}$ ¿qué es lo que hace que una red de colaboración funcione adecuadamente? Las habilidades blandas (soft) que son las que complementan a la tecnología (hard). Habilidades para liderar, gestionar equilibrar, trabajar en equipo y hacer posible la consecución de objetivos; autonomía, pasión pro actividad, autoliderazgo, integridad coherencia, capacidad de escucha activa, interés curiosidad, autenticidad, buena gestión del tiempo, responsabilidad personal y social, capacidad de reflexión, confianza, motivación, aprendizaje continuo, humildad, empatía (Roca, 2015). 
De acuerdo con las anteriores definiciones de las habilidades duras y habilidades blandas, se puede indicar que una no son excluyentes, sino que más bien son complementarias, de tal manera que no se puede caer en una división o dualismo en la formación por habilidades. En consecuencia, se puede caracterizar estas habilidades como se presenta en la tabla 1 .

Tabla 1. Síntesis de las características de las habilidades duras y blandas

\begin{tabular}{ll}
\hline \multicolumn{1}{c}{ Habilidades duras } & \multicolumn{1}{c}{ Habilidades blandas } \\
\hline Coeficiente intelectual & Habilidades no cognitivas \\
Conocimiento exacto & Relaciones interpersonales \\
Destrezas puntuales & Instrumentos emocionales \\
Medibles y calificables & Estrategias afectivas \\
De índole técnico & Estrategias sociales \\
& Aptitudes sociales \\
& Flexibilidad \\
& Adaptabilidad a la incertidumbre \\
& Liderazgo \\
& Trabajo en equipo \\
\hline
\end{tabular}

Fuente: Original de los autores.

Como se observa, el número de las características de las habilidades duras es menor respecto a las habilidades blandas. Sin embargo, las habilidades blandas han sido desplazadas en los proyectos educativos y se han relegado al currículo oculto, de tal manera que hoy en día las empresas y organizaciones están solicitando a las instituciones de educación y formadoras tener presente siempre las habilidades blandas como estrategia pedagógica para fomentar el éxito de las personas en formación. Esta situación es problémica en el contexto militar porque han enfatizado las habilidades duras en sus procesos formativos.

También se debe tener en cuenta que la designación de habilidades blandas y duras se fundamenta en la clasificación de ciencias duras, es decir, las ciencias exactas hijas de las matemáticas y de la física, y las ciencias blandas, consideradas como herederas de las humanidades y las ciencias sociales. Esta diferenciación generó un dualismo entre las ciencias duras y las ciencias blandas, de tal manera que quienes estudiaban las ciencias duras eran considerados seres humanos fríos, calculadores, medidos, exactos, estadísticos, entre otras consideraciones. Por su puesto, para el centro de educación formal era más fácil y sencillo evaluar su proceso formativo desde esta concepción, ya que sencillamente se hacía uso de la memoria, la repetición, el aprendizaje mecánico, el quiz, la previa, las preguntas de selección múltiple, 
entre otras estrategias evaluativas. Las habilidades duras se orientan a ejecutar acciones especializadas con precisión y un sistema riguroso, por ejemplo un guitarrista, un operario maquinista, entre otros.

En contraste, quienes estudiaban las ciencias sociales y humanidades eran considerados personas que pensaban el mundo y sus relaciones, que hacían uso de la hermenéutica, la descripción, la fenomenología de lo que sucedía en el mundo de la vida. Dado que estos aspectos son difíciles de evaluar en un proceso de educación formal, se recurrió entonces a estrategias de evaluación propias de las ciencias duras, las cuales son inadecuadas para las ciencias blandas.

Desde hace años, la discusión entre ciencias duras y ciencias blandas, así como entre habilidades duras y habilidades blandas ha sido superada en el campo teórico, pero desafortunadamente no ha sucedido lo mismo con el planteamiento de los proyectos educativos ni mucho menos con los proyectos de aula ${ }^{3}$. Por esta razón, a continuación se hace un acercamiento a las habilidades blandas, necesarias en un mundo que se ha comprometido con los diecisiete objetivos del desarrollo sostenible (ODS).

Las habilidades blandas están inmersas en el contexto de las relaciones interpersonales y resultan más complejas de planificar y evaluar. Por tanto, son incluidas en menor medida en los objetivos curriculares y dependen, en mayor medida, del ambiente que caracteriza el desarrollo de cada individuo y del contexto que condiciona los proyectos educativos de formación. En este orden de ideas, vale la pena preguntarse qué se entiende y cómo se forman las habilidades blandas en el contexto de la formación del militar. Para resolver este interrogante es necesario hacer un análisis juicioso y emprender acciones urgentes para que este tipo de formación no se quede rezagada ante las realidades actuales.

En los últimos años varios investigaciones en educación (Damasio, 2018; Duckworth, 2016; Goleman, 1995) han demostrado que las habilidades blandas resultan ser igual o, incluso, más importantes en los procesos de enseńanza-aprendizaje. Estas investigaciones son una invitación, desde la ciencia, a reconocer la relevancia de las habilidades que se habrían dejado por fuera de los objetivos curriculares por ausencia de instrumentos adecuados de medición y evaluación o por la consolidación de paradigmas según los cuales este tipo de habilidades eran parte de la personalidad y el carácter (no moldeable) del individuo. Por tal razón, dentro de la formación de los militares en el ámbito de la investigación es necesario encontrar

3 Cabe señalar que en este trabajo se hace referencia a estos aspectos para no perder de vista que las acciones educativas, así como los proyectos curriculares, deben estar inmersos y son resultado de una historia. 
un equilibrio pedagógico que permita el desarrollo simultáneo y escalonado de las habilidades blandas y duras. De esta manera, será posible garantizar que no solo se esté formando un buen investigador en la academia, sino un individuo que tenga la capacidad de transferir sus conocimientos y habilidades hacia otros contextos.

Para fomentar el desarrollo de las habilidades blandas en los proyectos educativos y curriculares en la formación del militar es necesario enmarcarlas y articularlas con los procesos de socialización y las dimensiones subjetivas e intersubjetivas. Asimismo, es fundamental estudiar estos procesos y dimensiones en el contexto específico de la educación militar para hacer una propuesta más adecuada sobre las habilidades blandas que debe desarrollar el militar para su desempeño profesional. No se trata de dar cursos o materias específicas, sino más bien de pensar un currículo que vaya más allá de las clases formales y en el cual el aula de clase integre la misma vida y los procesos sociales actuales. Con este propósito, el siguiente apartado hace una revisión de diversas fuentes que han recopilado información sobre la educación que reciben usualmente los militares y las habilidades que desarrollan en distintos programas de formación. Además evalúa el énfasis que hacen estos programas en la investigación y el desarrollo de las habilidades que corresponden a esta tarea.

\section{Educación militar con enfoque investigativo}

Para contextualizar este apartado es importante señalar que muchos avances tecnológicos han sido producto de los procesos de investigación que se realizan en el campo militar. No es desconocido que gracias a los ejércitos existen avances significativos y serios en el campo tecnológico como el internet, resultado del proyecto militar Arpanet. Por esta razón es necesario que los encargados de proponer y ejecutar los proyectos educativos y curriculares en el contexto militar tengan en cuenta que es importante desarrollar, potenciar y poner en práctica las habilidades duras y blandas — con especial énfasis en estas últimas-.

\section{La educación militar por competencias}

La formación militar tiene un fuerte énfasis en el desarrollo de habilidades relacionadas con la defensa, la estrategia y otras prácticas vinculadas al desarrollo de la labor militar. En ese sentido, el coronel Peláez (2008) plantea que los estudiantes requieren cuatro tipos de recursos para alcanzar el desarrollo de las habilidades requeridas: recursos humanos, materiales, financieros y de conocimiento o capital intelectual. Este último debe ser gestionado correctamente, de manera que 
se debe transmitir en el momento indicado y a las personas indicadas. Además, el autor señala que la correcta gestión de capital intelectual requiere estructuras que faciliten la sistematización de la información y la creación de conocimiento, así como procesos para su almacenamiento y difusión. Es decir, de acuerdo con Peláez (2008), si bien las habilidades duras son fundamentales en la formación del militar —aspectos que se deben articular con procesos cognitivos—, no se pueden desconocer las habilidades blandas, pues no es suficiente con saber, sino que también es necesario formar en el ser.

Peláez (2008) también resalta la importancia del conocimiento como motor de innovación y de transformación, ya que si se implementa de manera correcta se generan canales de retroalimentación y comunicación que permiten identificar oportunidades de mejora, lo cual resulta de gran importancia en el mundo actual por la gran velocidad en que se desarrollan los acontecimientos. Al respecto, Peláez (2008) plantea que si en años anteriores uno de los principales obstáculos para la investigación era la falta de información, ahora uno de los mayores desafíos es el exceso de información, ya que si se gestiona de manera incorrecta puede generar resultados inesperados.

Centrando esta reflexión en la discusión sobre las habilidades se encuentra que las condiciones actuales de acceso a la información están generando serios problemas en la gestión y la autogestión del conocimiento. Por lo tanto, se evidencia aún más la necesidad de planear, ejecutar y evaluar una formación holística e integral que complemente las dimensiones cognitivas propias de la formación militar con el desarrollo de las habilidades blandas.

Por su parte, Vargas, Valencia, Vanegas y Calcetero (2009) analizaron la evolución de los procesos educativos en la Escuela Militar de Cadetes "General José María Córdova” (ESMIC), reflexionando sobre la educación tradicional y contemporánea con respecto a la formación por competencias. Cabe recordar que la ESMIC fue creada en 1907 para formar oficiales del Ejército Nacional en ciencias militares, de tal manera que los diferentes planes de estudio que ofrece buscan formar hombres y mujeres profesionales, éticos, con valores cívicos y morales de respeto por los principios democráticos y por el Estado social de derecho planteados en la Constitución nacional.

De acuerdo con Vargas et al. (2009), el enfoque pedagógico para la formación militar ha atravesado por tres etapas: la primera tuvo lugar alrededor de 1970, cuando se utilizó un enfoque tradicional (método lancasteriano), caracterizado por una relación extrema de dominación y autoridad en la que el estudiante se acogía, exclusivamente, al mandato del docente. En la segunda etapa, que transcurre 
durante los años 1980, la ESMIC tuvo un giro hacia el enfoque constructivista, en el cual el papel del docente empezó a ser de facilitador y acompañante del proceso del estudiante. Finalmente, la tercera etapa comenzó con la década de 1990 y se caracterizó por el predominio del enfoque holístico, el cual integraba diferentes elementos de los enfoques utilizados anteriormente con el objetivo de fortalecer la enseñanza-aprendizaje (Vargas et al., 2009).

Recientemente, en el panorama educativo nació un enfoque que propone al docente como un mediador y facilitador en el proceso, y que busca formar individuos de transformación competente en la sociedad. En este nuevo contexto, la vía para generar conocimiento es el autoaprendizaje significativo, es decir, aprender a aprender. Por esta razón la ESMIC contempla todos estos enfoques y empezó a implementar también modelos de formación por competencias con el fin de ofrecer educación integral a los militares. Al respecto, los autores consideran que es necesario articular los distintos elementos de los enfoques tradicionales y contemporáneos que históricamente han aportado componentes valiosos a la formación.

Asimismo, Vargas et al. (2009) resaltan que las transformaciones en los enfoques pedagógicos en Colombia han sido influidas por los contextos históricos, culturales, políticos, etc. También señalan que las transformaciones en estos procesos educativos han sido muy lentos y poco progresivos porque, argumentan, en Colombia predomina un enfoque conductista. En el enfoque tradicional, el contenido curricular contiene una serie de habilidades que tienen que ser aprendidas, ejecutadas y medidas, mientras que el enfoque conductista se centra en las condiciones de aprendizaje (Vargas et al., 2009). Para los autores, en Colombia este enfoque educativo no ha permitido que los egresados desarrollen capacidades analíticas, investigativas, científicas, críticas, metacognitivas, etc. Sostienen que la educación tradicional se centra en enseñar el desempeño de habilidades que serán evaluadas

en forma cuantitativa siempre midiendo rigurosamente el desempeño con estándares absolutos [...] la educación tradicional ha dejado que el proceso educativo se centre en la formación de seres humanos con unas habilidades vocacionales para que desempeñen cargos y labores en función del trabajo, de la producción y de la simple transmisión de conocimientos de unos a otros, dejando de lado la construcción, la producción y la creación de nuevo conocimiento. (Vargas et al., 2009, p. 26)

En esta misma línea, dado que el modelo tradicional cognitivo ha caracterizado la formación militar, se evidencia que persiste el vacío de las habilidades blandas, de manera que es necesario resignificarlo de acuerdo con los procesos sociales actuales.

Específicamente, en la ESMIC el enfoque tradicional sigue teniendo gran relevancia, aunque con el tiempo se han generado cambios acordes con los nuevos 
modelos educativos. Por esto, aunque la Escuela Militar ha enmarcado su proyecto educativo en el enfoque constructivista, el peso que tiene el enfoque tradicional y la formación por competencias sigue entorpeciendo este proceso. Esto quiere decir que cuando se analizan las tendencias de transformación en el quehacer educativo militar, se encuentra que las habilidades duras siguen siendo el centro y foco de interés formativo, de manera que se dejan de lado las habilidades blandas.

Respecto al enfoque de enseñanza por competencias, Vargas et al. (2009) plantean perspectivas más integrales elaboradas por otros autores (competencias desde la complejidad). Así, describen las competencias como "capacidades, habilidades y destrezas que desarrolla el educando para responder de formas particulares y con intencionalidades definidas en la solución de problemas en situaciones específicas" (Vargas et al., 2009, p. 31). Es decir que el concepto de competencia no se debe entender como competitividad en el sentido laboral, sino como el saber, saber ser, saber saber y saber hacer que cada individuo ha desarrollado con base en su experiencia. Asimismo, se debe tener en cuenta que estos conocimientos son únicos, pues han sido aprendidos de un modo notable, analizados y asumidos críticamente de un modo particular. Ahora bien, si las habilidades se abordan como skills, entonces se debe aceptar que habilidad es sinónimo de competencia, pero no es objeto de este escrito discutir cuál debe ser su definición etimológica, sino más bien su significado dentro del proceso de formación y educación.

En este sentido, se requiere la profundizar en el proceso de enseñanza-aprendizaje, lo cual implica transformar las diferentes etapas: planificación, ejecución, evaluación y seguimiento dentro del aula. Para lograrlo es indispensable que se cuente con un diseño claro del currículo que se va a implementar en el programa educativo, el cual debe tener como propósito principal potencializar las habilidades, las competencias, las destrezas y los desempeños, esto quiere decir, hacer una articulación real entre las habilidades blandas y las habilidades duras.

Este currículo debe enfatizar la formación en investigación, para lo cual tiene que crear estrategias que lleven a los estudiantes a desarrollar las habilidades simples que necesitan para adquirir las habilidades complejas que demanda la investigación dentro y fuera del contexto académico. Esta perspectiva implica formular un currículo que considere el proceso de "desaularización", es decir, que el aula de clase sea la sociedad, la realidad de los contextos, los procesos sociales coyunturales. Del mismo modo, es indispensable consolidar un proyecto educativo que tenga en cuenta la naturaleza holística del individuo y que busque desarrollar habilidades que no se concentren exclusivamente en el quehacer del militar, sino que más bien tenga como centro de la formación una concepción antropológica y sociológica. 
En otras palabras, un proyecto educativo que repiense y resignifique los medios, diferenciándolos de los fines de la educación militar.

Con ese objetivo, las instituciones han adoptado el enfoque de la enseñanza por competencias desde la comprensión de la complejidad del individuo (Vargas et al., 2009), desde el cual se definen tres competencias esenciales. En primer lugar está el saber ser, que se refiere al desempeño humano frente a un problema específico y que tiene tres elementos: sensibilización, personalización de la información y cooperación. En este saber influyen de manera positiva elementos afectivos y motivacionales si se manejan bien, pues pueden generar apertura mental, interés, disposición, etc. La segunda competencia es el saber conocer, que está relacionado con crear habilidades y estrategias para aprender y comprender sin tener que memorizar. Incluye el análisis crítico, la indagación sistemática, la clasificación de pensamientos, la articulación de conocimientos con experiencias, el uso del conocimiento en contexto, en la realidad. Finalmente, el saber hacer asume que "la mejor forma de aprender algo es haciéndolo" y que en el error se logra la toma de conciencia.

En general, la relevancia de este capítulo yace en la actualización del enfoque pedagógico que caracteriza el proceso de aprendizaje-enseńanza en el ámbito militar, el cual permite a los estudiantes desarrollar las múltiples habilidades que exigen los contextos diversos que enfrentan, al tiempo que se fortalecen las habilidades que requieren para ejercer la labor estratégica militar. De acuerdo con Vargas et al. (2009), si bien los procesos educativos basados en competencias desde una visión de complejidad responden a diversas situaciones, también se debe señalar que tienen limitaciones porque enfatizan aspectos propios de las habilidades duras; es más, en este enfoque se evidencia una confusión entre las competencias y las habilidades debido a que se centra en aspectos sociocognitivos. Por lo tanto, es importante destacar que en el campo de la educación formal, particularmente en los escenarios de la educación universitaria, se debe hacer énfasis en la formación para la investigación y potenciar las habilidades necesarias para tal fin.

\section{Habilidades para la investigación}

Si bien es cierto que es fundamental comprender al individuo desde una perspectiva holística e integral, dimensión desde la cual se reconoce la complejidad de las habilidades (duras y blandas), así como la importancia de una educación que promueva el desarrollo de habilidades de fácil transferencia a diferentes contextos, incluyendo el ámbito de la investigación, también es indispensable reconocer y re-afirmar con claridad cuáles son las habilidades requeridas para formar seres 
humanos que tengan disposición hacia la investigación y que puedan generar este tipo de labores desde su papel como militares.

De acuerdo con Martínez y Márquez (2014), las habilidades investigativas pueden clasificarse en cuatro categorías. La primera está conformada por las habilidades básicas de la investigación, que son aquellas propias de la ciencia particular y de la metodología de la investigación. En ese sentido, se consideran necesarios los conocimientos básicos requeridos para poder desarrollar una investigación en un área específica del conocimiento. Por ejemplo, para poder realizar una investigación sobre neuroeducación, es indispensable tener conocimientos avanzados en neurología, neurobiología o educación. Al mismo tiempo, se requieren conocimientos en metodología investigativa (cuantitativa, cualitativa, mixta, etc.). Este tipo de habilidades tienen una naturaleza transdisciplinar y están vinculadas a paradigmas epistemológicos que van más allá de una formación meramente disciplinar. Además de esto, se requieren otro tipo de habilidades que tienen que ver con las relaciones multidisciplinarias que se establecen en el currículo, habilidades de lógicas de pensamiento como: analizar, sintetizar, comparar, abstraer, observar, describir, definir, caracterizar, explicar, demostrar, ordenar, modelar. Estas son habilidades complejas que se caracterizan porque corresponden a un ejercicio cognitivo de interrelación entre habilidades del pensamiento, las cuales son necesarias en la formación del militar.

La segunda categoría se refiere a las habilidades que implican la capacidad de problematizar, teorizar y contrastar. Este tipo de habilidades, de acuerdo con la categorización ofrecida anteriormente, se pueden reconocer como complejas, ya que requieren del desarrollo de diversas habilidades sencillas como explorar, conocer, investigar, indagar, recolectar datos, predecir, etc. Así, es indispensable que para lograr la maestría de estas habilidades se haga un escalonamiento previo que contemple el proceso de aprendizaje como un todo complejo que busca un objetivo específico y que tiene un propósito particular. Por tanto, es indispensable desarrollar programas educativos que contemplen como parte de su fin último la formación de estudiantes con espíritu investigativo y disposición hacia las labores de investigación en múltiples ámbitos. No se trata de formar militares científicos, encerrados en un laboratorio, sino que más bien se forme un militar que tenga las herramientas necesarias para saber liderar la transformación y resolución de problemas que se le presenten en un determinado contexto.

La tercera categoría agrupa las habilidades de percepción, instrumentales, de reflexión, etc. Desde la perspectiva de Ritchhart (2011), este tipo de habilidades podrían denominarse como habilidades de pensamiento y son la base para el desa- 
rrollo cognitivo y metacognitivo. Este tipo de habilidades permite a los estudiantes la construcción individual y colectiva del conocimiento, así como el proceso de pensar sobre el pensamiento y sobre los procesos de aprendizaje. No obstante, pese a que son poco valoradas en las culturas institucionales, de acuerdo con Martínez y Márquez (2014) son la espina dorsal del proceso de investigación. Al respecto, Perkins (2014) sostiene que no son solo relevantes en el ámbito de la investigación, sino también en el desarrollo de individuos que estén preparados para los drásticos y rápidos cambios que caracterizan el mundo actual. En gran medida, esa preparación que se hace desde el desarrollo del pensamiento (y en últimas, la consolidación del pensamiento crítico), implica una preparación extrema para la resolución de problemas, conflictos y la adaptación a nuevos contextos, lo cual está implícito e inmerso en la labor investigativa. Esto quiere decir que cuando se formula y proyecta un currículo centrado en la formación para la investigación y desde la potenciación de las habilidades, se rompe con el dualismo de habilidades blandas y habilidades duras, de tal forma que se formula un proyecto educativo integral y holístico, reto que tiene la formación militar en estos momentos históricos.

Finalmente, la cuarta categoría hace referencia a las habilidades investigativas que requieren mayor integración con el desarrollo propio de los conocimientos que se abordan en un programa profesional (pregrado). En este sentido, las habilidades desarrolladas en torno a la investigación empiezan a vincularse exclusivamente al área profesional en el que se desempeña un individuo. Así, se ha planteado que existen cinco habilidades básicas en esta categoría, que tienen naturaleza relacional y que se ponen en práctica en función de la resolución de problemas propios de un área del conocimiento: modelar, obtener, procesar, comunicar y controlar. Así, desde esta perspectiva la resolución de problemas se centra de nuevo en las habilidades duras, aspecto que torna la investigación como una labor fría y calculadora, que no le permitiría flexibilizarse y adaptarse a los principios de la incertidumbre ni la complejidad.

Por su parte, Josefina Balbo (2015) ha establecido una serie de competencias que deberían fortalecerse desde el pregrado para generar profesionales con perfil de investigación. En primer lugar señala la integración del estudiante en un ámbito universitario. Segundo, la disposición para el trabajo y la construcción colectiva. Tercero, la disposición para identificar, formular y resolver problemas. Cuarto, la disposición para desarrollar procesos de comunicación efectiva. Quinto, fortalecer acciones éticas y la responsabilidad social. Sexto, la capacidad de actualización permanente y el emprendimiento. Séptimo y último, la capacidad para generar y difundir conocimiento. Al hacer un acercamiento a este planteamiento se evidencia 
nuevamente que la formación para la investigación persiste e insiste en que se haga énfasis en un proceso frío y racional que no tiene en cuenta las habilidades blandas, sino que solamente se preocupa por formar investigadores competentes, olvidando su carácter de ciudadanos del mundo. La deuda de las habilidades para la investigación es evidente, de modo que salta a la vista la necesidad de implementar un sistema educativo y curricular que sea integral y holístico.

Con base en esta síntesis se puede afirmar que este apartado ha permitido reconocer la existencia de una serie de habilidades que se relacionan exclusivamente con la investigación. Atendiendo a un tipo de categorización y clasificación flexible, es posible establecer, en primera instancia, que las habilidades propias de la investigación se relacionan con múltiples áreas del conocimiento y que requieren de un contexto significativo de aprendizaje en el que se permita un desarrollo transversal de habilidades duras, pero que tiene retos y aspectos pendientes con las habilidades blandas.

\section{Habilidades de investigación en la educación militar en Colombia}

Hasta el momento, el estudio sobre las habilidades investigativas en las universidades o escuelas militares en Colombia permanece reducido y hay poco conocimiento en términos del desarrollo de estas habilidades en las escuelas. Específicamente en este ámbito, Arana et al. (2011) hicieron un estudio para conocer las habilidades investigativas de los estudiantes de la ESMIC, para lo cual evaluaron un número amplio de investigaciones para tesis de grado de la Facultad de Ciencias Militares de la institución.

Para analizar las tesis los autores se basaron en el enfoque Ciencia, Tecnología y Sociedad (CTS). Este enfoque desarrolla la concepción integral del conocimiento y plantea que no es suficiente comprender el mundo basado exclusivamente en métodos y modelos, sino que también hay que integrarlos y unificarlos con la sociedad y la profesión. A su vez considera necesario el desarrollo del pensamiento creativo y crítico que busque la evaluación de los contextos para generar soluciones que aporten valor a problemas que tienen impacto negativo en la sociedad. En ese sentido, Arana et al. (2011) aseveran que es necesario tener una perspectiva de integralidad, a pesar de que el análisis se aborde desde una profesión única.

Ahora bien, con respecto a las competencias y las habilidades, los autores plantean que

[l]as capacidades, habilidades y actitudes son solo predisposiciones en la persona; las competencias, por el contrario, se refieren a las actuaciones, a la actividad, a la acción de esta en la sociedad, en el contexto. Por tanto, en las competencias 
está presente la relación individuo-sociedad; puesto que dichas predisposiciones se manifiestan en la actividad profesional y en la vida social, de ahí que las competencias, desde lo pedagógico, deben formarse en el aprender, pero haciéndose desde la práctica. (Arana et al., 2011, p. 156)

Asimismo, plantean que la implementación del aprendizaje por competencias como enfoque privilegiado en la Escuela permite el desarrollo consciente y con propósito de habilidades y actitudes. Esto hace posible el desarrollo de disposiciones que permitirán a los estudiantes transferir lo que han aprendido a múltiples contextos. Es decir, es el elemento clave para hacer que el aprendizaje tenga un impacto positivo en la sociedad que está más allá de las aulas universitarias o escolares. En ese sentido, se plantea un contexto que permita el desarrollo de cuatro competencias fundamentales: saber, saber ser, saber hacer y saber convivir. Estas cuatro competencias abarcan habilidades y destrezas que se relacionan con los principios, valores y virtudes que configuran el perfil del militar. Sin embargo, se evidencia de nuevo que se hace más énfasis en el nivel cognitivo de las habilidades.

Ahora bien, como parte esencial del desarrollo de la investigación y para determinar la muestra, los autores analizaron los registros de trabajos de grado entre 1999 y 2009, periodo en el que hubo variaciones importantes en el número de trabajos de grados realizados. Arana et al. (2011) explican que esto se debe a dos razones: primero, cambios en las políticas para realizar los trabajos de grado, pues inicialmente estos se realizaban individualmente, pero posteriormente, con el aumento significativo del número de alumnos, hubo un cambio de política para que los estudiantes pudieran trabajar en grupos, que en muchas ocasiones eran de hasta cuatro personas. La segunda razón es el aumento en el número de alumnos graduados, que llegaron a ser hasta ochocientos por año debido a la reducción del tiempo de estudio a tres años.

Respecto a los trabajos en sí, los autores encontraron que hay competencias básicas que no se ven reflejadas en las investigaciones de grado, lo cual evidencia que no ha habido un desarrollo adecuado de estas competencias durante el proceso de aprendizaje. En primera instancia, lo autores consideran que hay una ausencia de curiosidad en la exploración investigativa. Segundo, establecen que la capacidad de búsqueda de información y extensión de lo que se conoce es limitada y está reducida a lo que se ofrece en la web. Tercero, hallaron que los estudiantes tienen poco interés por trabajar en problemáticas no resueltas por diferentes áreas del conocimiento, de manera que hace falta mayor desarrollo de las habilidades relacionadas con la innovación y la creatividad. Cuarto, no encontraron evidencias de un esfuerzo por reconocer el estado del arte ni por construir sobre los avances de deter- 
minado ámbito. Quinto, Arana et al. (2011) notaron una ausencia de comunidad científica, espacios de socialización y construcción colectiva del conocimiento, por tanto, no hay publicaciones científicas o espacios de divulgación oral de los resultados de las investigaciones realizadas.

Se presenta, entonces, una combinación de diferentes habilidades (blandas y duras) que se deberían desarrollar en el proceso de enseñanza-aprendizaje durante la educación básica, secundaria y profesional. Este proceso debe responder a una planificación inicial que escalone los objetivos relacionados con el desarrollo de habilidades y conocimientos para alcanzar la maestría en el cumplimiento de objetivos complejos. En este sentido, se puede afirmar que existe una falencia inicial en el sistema educativo que debe reparar la ESMIC para garantizar que las habilidades investigativas se estén incluyendo de forma transversal en el proceso de formación profesional del militar.

\section{Estrategias pedagógicas para desarrollar habilidades investigativas}

A través de los apartados anteriores es posible entrever que existe una serie de habilidades que permitirán a los militares formarse con un perfil profesional que apunta hacia la investigación (López, 2001). Estas habilidades se han categorizado en competencias que corresponden con el modelo educativo predominante en Colombia y que son transversales al saber, al ser y al hacer. En este sentido, es posible afirmar que más allá de habilidades inherentes a la investigación (Machado et al. 2008), están relacionadas con la formación de ciudadanos activos del mundo con suficiente flexibilidad para adaptarse a un mundo de rápidos cambios y crecientes desafíos para la humanidad.

Asimismo, se ha evidenciado que ha habido poco desarrollo de estas habilidades a pesar de los esfuerzos de las escuelas y las universidades militares por utilizar estrategias pedagógicas innovadoras y cambiar antiguos enfoques educativos. En este sentido cabe formularse la siguiente pregunta: ¡la formación en habilidades para la investigación y para responder a los retos del mundo se debe realizar desde estrategias pedagógicas y didácticas? Es por ello que resulta fundamental reflexionar cuáles son las estrategias formativas pertinentes que se deberían articular en el proceso de enseñanza-aprendizaje de los militares para desarrollar las habilidades, tanto duras como blandas, que requiere la investigación. En respuesta a esta necesidad, a continuación se presentan algunas alternativas que se pueden implementar en la formación del militar. 


\section{Pensamiento de diseńo y makers-centers}

Estas propuestas buscan sacar del aula de clase el proceso de aprendizaje y articularlo con la resolución de problemas reales, lo cual resulta bastante apropiado en el contexto de la educación militar porque parte de la naturaleza de la labor militar es el servicio a la ciudadanía. Adicionalmente, estas pedagogías permiten desarrollar una habilidad blanda crucial en el perfil del investigador que es la empatía. El desarrollo de la empatía permite consolidar respuestas y soluciones innovadoras que apuntan a resolver problemas de poblaciones reales, reconociendo sus contextos, necesidades, intereses y valores. En este sentido, se empiezan a trabajar diversas habilidades simples que, posteriormente, permitirán configurar habilidades complejas para la consecución de las labores investigativas.

Específicamente, el pensamiento de diseño contempla diversas fases —empatizar, definir, idear, prototipar y testear - que permite a los estudiantes familiarizarse con el método científico y desarrollar diversas habilidades simples relacionadas con la investigación. Entre ellas se encuentra definir, reflexionar, conectar, predecir, recolectar evidencia, concluir, contrastar, evaluar, etc. Del mismo modo, los makers-centers buscan que los estudiantes desarrollen habilidades a través de la práctica.

Ambas estrategias buscan que el estudiante desarrolle la agencia en el aprendizaje, entendida como una disposición del individuo a tomar decisiones a partir de la autonomía y la decisión individual basada en criterios morales e intelectuales que superan las constricciones sociales establecidas por un contexto establecido. Esto le permitirá superar las limitaciones que podrían evitar la consolidación de respuestas innovadoras.

\section{Aulas invertidas}

Esta estrategia pedagógica permite dar un giro total a las aulas tradicionales, en cuanto busca que los estudiantes indaguen, exploren y preparen previamente, de forma individual o colectiva, los conceptos y teorías que se van a trabajar durante la clase. Esto permite que el tiempo de la clase se destine a desarrollar proyectos en los cuales se ponga en práctica el conocimiento adquirido previamente. Esto incluye múltiples proyectos y tareas: debates, experimentos, experiencias fuera de la institución y en contacto directo con comunidades externas.

Esta estrategia permite que en su proceso de trabajo personal, individual o colectivo fuera de las aulas, los militares trabajen en el desarrollo de habilidades investigativas y de indagación. Con ello, desarrollarán no solo habilidades simples relacionadas con tareas de investigación como la indagación, la observación, la recolección de datos, la información, etc., sino que además pondrán a prueba su 
criterio para seleccionar información e implementar estrategias para reconocer la veracidad de la información, las fuentes y los datos, así como la habilidad para explorar el mundo con curiosidad a partir de lineamientos claros establecidos por un docente que ve transformado su papel de facilitador a mentor.

\section{Conclusión}

Si bien es cierto que la educación militar ha desarrollado una serie de estrategias y enfoques pedagógicos que permiten el desarrollo transversal de habilidades blandas y duras, aún hay camino por recorrer para establecer procesos y estrategias pedagógicas que garanticen el desarrollo de estas habilidades orientadas hacia la investigación. Del mismo modo, es necesario hacer un escalonamiento planificado acorde con el diseño del programa educativo que permita el desarrollo procesual de habilidades simples para alcanzar el objetivo final de la consolidación de habilidades complejas. Estas últimas, cabe recordar, son las que finalmente le permitirán al militar configurar un perfil profesional que incluya la investigación.

Para tal razón es indispensable repensar no solo el proceso de planificación de los programas educativos de los militares, sino considerar nuevas estrategias pedagógicas que garanticen el desarrollo de habilidades blandas, como la empatía o el manejo emocional, y de habilidades duras relacionadas con la investigación. Del mismo modo, requerirá una aproximación transversal inter- y transdisciplinaria que permita desarrollar las competencias del saber, saber ser y saber hacer. Por ello, se deben considerar estrategias pedagógicas como el pensamiento de diseño, los makers-centers y las aulas invertidas para que los militares logren un acercamiento directo con el aprendizaje desde la práctica y el contacto constante con las ciudadanías (a quienes, en últimas, servirán).

\section{Referencias}

Alex, K. (2016). Soft skills, know yourself and know the world. Nueva Delhi: S. Chand \& Company.

Arana, M., Bonilla, G., Fernández, E., López, C., Ortiz, E., \& Fletscher, L. (2011). Las competencias de investigación presentes en los trabajos de grado realizados en la Facultad de Ciencias Militares durante los años 2008 y 2009. Revista Cientifica General José María Córdova, 9(9), 149-186.

Arroyo Tovar, R. (2012). Habilidades gerenciales: desarrollo de destrezas, competencias y actitud. Bogotá. D. C.: Ecoe.

Badaró, M. (2009). Militares o ciudadanos. La formación de los ofciales del Ejército Argentino. Buenos Aires: Prometeo. 
Balbo, J., Pacheco, M. y Rangel, Z. (2015). Medición de las competencias investigativas en los docentes adscritos al Departamento de Ciencias Sociales de la Universidad Nacional Experimental del Táchira. Aibi Revista de Investigación, Administración E Ingeniería, (3) 2, 27-36.

Bassi M., Busso, M., Urzua, S. \& Vargas, J. (2012). Desconectados: habilidades, educación y empleo en América Latina. Washington: Banco Interamericano de Desarrollo. Recuperado de http:// idbdocs.iadb.org/wsdocs/getdocument.aspx?docnum=36723125

Damasio, A. R. (2018). The strange order of things: life, feeling, and the making of cultures. Nueva York: Pantheon Books.

Duckworth, A. (2016). Grit: the power of passion and perseverance. Nueva York: Scribner.

Goleman, D. (1995). Emotional intelligence. Nueva York: Bantam Books.

Lagos García, C. (2012). Aprendizaje experiencial en el desarrollo de habilidades "blandas" (Tesis de pregrado en Trabajo Social). Universidad Alberto Hurtado, Santiago de Chile.

López, L. (2001). El desarrollo de las habilidades de investigación en la formación inicial del profesorado de química. Cienfuegos: Universidad de Cienfuegos Carlos Rafael Rodríguez.

Machado, E., Montes de Oca Recio, N., \& Mena, A. (2008). El desarrollo de habilidades investigativas como objetivo educativo en las condiciones de la universalización de la educación superior. Revista Pedagogía Universitaria, 13 (1), 156-180. http://revistas.mes.edu.cu/ PedagogiaUniversitaria/articulos/2008/numero/189408108.pdf

Martínez, D., \& Márquez, D. (2014). Las habilidades investigativas como eje transversal de la formación para la investigación. Tendencias pedagógicas, (24), 340-360.

Moreno, M. (2005). Potenciar la educación. Un currículum transversal de formación para la investigación. Revista Iberoamericana sobre Calidad, Eficacia y Cambio Educativo, 3 (1), 520-540. http:// dialnet.unirioja.es/servlet/articulo?codigo=1130331

Mujica, J. (2015). Habilidades blandas [documento en línea]. Recuperado de https://educrea.cl/ wp-content/uploads/2016/02/DOC-habilidades-blandas.pdf

Peláez, A. (2008, Agosto). Gestión del conocimiento: factor clave para generar capacidades de defensa. Revista del IMES, 9 (14), 17-31.

Perkins, D. (2014). Future wise: educating our children for a changing world. San Francisco: Jossey Bass.

Repetto, E. (Dir.). (2009). Formación en competencias socioemocionales. Madrid: La Muralla.

Roca, R. (2005). Knowmads: los trabajadores del futuro. Bogotá, D. C.: Lid.

Ritchhart, R. (2011). Making thinking visible: how to promote engagement, understanding, and independence for all learners. San Francisco: Jossey Bass.

Shaffer, D. (2002). Desarrollo social y de la personalidad. Madrid: Paninfo.

Tobón, S. (2009). Formación basada en competencias. Pensamiento complejo, diseño curricular y didáctica. Bogotá, D. C.: Ecoe.

Valdevenito M. J. (2013). Habilidades blandas, fundamentales para el desarrollo personal. Santiago de Chile: Universidad Alberto Hurtado.

Vargas, M., Valencia, M., Vanegas, N., \& Calcetero, R. (2009). Una mirada de la formación por competencias para los procesos académicos de la Facultad de Ciencias Militares de la Escuela Militar de Cadetes “General José María Córdova”. Revista Educación y Desarrollo Social, 3 (1), 21-36. 